\title{
Analysis of the Pathological Examination Results of 100 Colon Polyps Cases after EMR or ESD Operation
}

\author{
Shibao Song ${ }^{*}$, Weigang Liu ${ }^{1 *}$, Dong Wang ${ }^{1}$, Chuanxin Zou ${ }^{2 \#}$ \\ ${ }^{1}$ The Second Clinical Medical College, Yangtze University, Jingzhou, China \\ ${ }^{2}$ Department of Gastroenterology, Jingzhou Central Hospital, The Second Clinical Medical College, Yangtze University, \\ Jingzhou, China \\ Email: "1559734687@qq.com
}

How to cite this paper: Song, S.B., Liu, W.G., Wang, D. and Zou, C.X. (2018) Analysis of the Pathological Examination Results of 100 Colon Polyps Cases after EMR or ESD Operation. Yangtze Medicine, 2, 186-191.

https://doi.org/10.4236/ym.2018.23020

Received: July 27, 2018

Accepted: August 27, 2018

Published: August 30, 2018

Copyright ( 2018 by authors and Scientific Research Publishing Inc. This work is licensed under the Creative Commons Attribution International License (CC BY 4.0).

http://creativecommons.org/licenses/by/4.0/

\begin{abstract}
Aim: To study the characteristics of EMR (endoscopic mucosal resection) or ESD (endoscopic submucosal dissection) postoperative pathological examination of colonic polyps in different age groups, and the difference of pathological examination after EMR or ESD operation. Methods: A retrospective analysis was made of the EMR or ESD postoperative pathological data of 100 cases of colonic polyps patients in the Jingzhou Central Hospital digestive internal medicine. The time is from March 2016 to March 2018. Results: In 100 cases of EMR or ESD operation, there are 65 cases of young and middle-aged group. Pathological examination results include: 34 cases of adenomatous polyp, 18 cases of inflammatory polyp, 11 cases of benign tumors, 1 case of malignant tumor, 1 case of hyperplastic polyp. There are 35 cases of elderly group. Pathological examination results include: 27 cases of adenomatous polyp, 2 cases of inflammatory polyp, 2 cases of benign tumors, 4 cases of malignant tumor. 71 cases of complete EMR operation include: 51 cases of adenomatous polyp, 17 cases of inflammatory polyp, 3 cases of benign tumors; 29 cases of complete ESD operation include: 10 cases of adenomatous polyp, 3 cases of inflammatory polyp, 10 cases of benign tumors, 5 cases of malignant tumor, 1 case of hyperplastic polyp. Conclusion: For the pathological examination results after EMR or ESD operation, the proportion of inflammatory polyps in the young and middle-aged group was greater than that in the elderly group. The proportion of adenomatous polyps in the elderly group was larger than that in the young and middle-aged group. The proportion of adenomatous polyps in EMR was greater than that of ESD. The proportion of benign tumors and malignant tumors in ESD was greater than that of EMR. The difference has statistical significance.
\end{abstract}

${ }^{\star}$ Contributed equally. 


\section{Keywords}

Colon Polyps, Endoscopic Mucosal Resection, Endoscopic Submucosal

Dissection, Pathological Examination Results

\section{Introduction}

With the development of gastrointestinal endoscopy, EMR and ESD are widely used methods in treatment of gastrointestinal polyps disease [1]. EMR refers to the injection of drugs in the submucosa of the lesion to form a liquid pad, and then cut off a chunk of mucous tissue. EMR avoids the defects of the traditional methods that the removal of mucosal biopsy tissue samples is too small and many cases have failed to make a correct diagnosis. It is an effective and reliable method for the treatment of precancerous lesions and early cancer. It has become one of the first choices for diagnosis and treatment of early cancer and critical lesions. The advantage is that it can increase the area and depth of resection, and achieve a radical cure. It is mainly suitable for the resection of partial sessile polyps, flat or shallow sunken type polyps, leiomyoma, early cancer (including esophagus, stomach, colon early cancer). EMR operation is safe and reliable, with fewer complications. ESD is a new therapeutic method in recent years and is also a promising technique for clinical applications. To allow more early gastrointestinal cancer to be completely resected under endoscopy, the pain of the laparotomy and the removal of the organ can be avoided. This study retrospectively analyzed the pathological examination results of 100 cases of colonic polyps after EMR or ESD operation in the evaluation of EMR and ESD postoperative pathological examination characteristics.

\section{Object and Methods}

First we need to claim that this investigation was approved by the Jingzhou central hospital ethics committee. We collect 100 patients in March 2016 to March 2018 in the Jingzhou center hospital digestive internal medicine, which using colonoscopy colon polyps was observed. The types of polyps that conform to the standard include: lateral developmental polyps, sessile or rebagliati polyps, polyp canceration without infringing submucosa (In combination with endoscopic ultrasonography to observe). EMR operation was used which diameters larger than $0.5 \mathrm{~cm}$ and less than $2.0 \mathrm{~cm}$. ESD operation was used for colonic polyps with a diameter greater than $2.0 \mathrm{~cm}$. Patients with severe cardiopulmonary disease, hemopathy, coagulation dysfunction and diseased elevation negative are excluded. All patients were given preoperative consent before colonoscopy and treatment. Both surgical treatments use endoscopic with EMR or ESD in our hospital endoscopy center. After the operation, lesion resection complete and check in the pathological department. All of the research objects are: the male 55 cases, female 45 cases, EMR operation 71 cases, ESD operation 29 cases. Young and middle-aged group (Less than 60 years of age) is 65 cases; elderly group 
(Greater than or equal to 60 years of age) is 35 cases. Referring all of the pathological examination results after the operation, EMR and ESD postoperative pathological examination characteristics are analyzed with a retrospective method.

\section{Statistical Analysis}

Using SPSS23.0 statistical software for statistical analysis, the data analysis using the $\chi^{2}$ test or Fisher's exact probability method of four table data, $\mathrm{P}<0.05$ for the difference is statistically significant.

\section{Results and Conclusion}

65 cases of young and middle-aged group completed EMR or ESD operation, pathological examination results include: 34 cases of adenomatous polyp (52.31\%), 18 cases of inflammatory polyp (27.69\%), 11 cases of benign tumors (16.92\%), 1 case of malignant tumor (1.54\%), 1 case of hyperplastic polyp (1.54\%); 35 cases of elderly group completed EMR or ESD operation, pathological examination results include: 27 cases of adenomatous polyp (77.14\%), 2 cases of inflammatory polyp (5.71\%), 2 cases of benign tumors (5.71\%), 4 case of malignant tumor (11.43\%), 0 case of hyperplastic polyp (0.00\%). Among them, adenomatous polyp elderly group share is greater than the young and middle-aged group, the difference is statistically significant $(\mathrm{P}<0.05)$. Inflammatory polyp young and middle-aged group share is greater than the elderly group. The difference is statistically significant $(\mathrm{P}<0.05)$. Specific results are shown in Table 1.

Among 71 cases completed EMR operation, pathological examination results include: 51 cases of adenomatous polyp (71.83\%), 17 cases of inflammatory polyp (23.94\%), 3 cases of benign tumors (4.23\%), 0 case of malignant tumors (0.00\%), 0 case of hyperplastic polyp (0.00\%); among 29 cases completed ESD operation, pathological examination results include: 10 cases of adenomatous polyp (34.48\%), 3 cases of inflammatory polyp (10.34\%), 10 cases of benign tumors $(34.48 \%), 5$ cases of malignant tumor (17.24\%), 1 case of hyperplastic polyp (3.45\%). Among them, adenomatous polyp in EMR operation group share is greater than the ESD operation group, the difference is statistically significant $(\mathrm{P}<0.01)$. Benign tumors and malignant tumor in ESD operation group share is greater than the EMR operation group. The difference is statistically significant $(\mathrm{P}<0.01)$. Specific results are shown in Table 2.

Table 1. EMR, ESD operation pathological examination results among all age groups case (\%).

\begin{tabular}{cccc}
\hline Pathological examination results & Young and middle-aged group & Elderly group & P-value \\
\hline adenomatous polyp & $34(52.31)$ & $27(77.14)$ & $<0.05$ \\
inflammatory polyp & $18(27.69)$ & $2(5.71)$ & $<0.05$ \\
benign tumors & $11(16.92)$ & $2(5.71)$ & $>0.05$ \\
malignant tumors & $1(1.54)$ & $4(11.43)$ & $>0.05$ \\
hyperplastic polyp & $1(1.54)$ & $0(0.00)$ & $>0.05$ \\
\hline
\end{tabular}


Table 2. EMR, ESD operation pathological examination results case (\%).

\begin{tabular}{cccc}
\hline Pathological examination results & EMR & ESD & P-value \\
\hline adenomatous polyp & $51(71.83)$ & $10(34.48)$ & $<0.01$ \\
inflammatory polyp & $17(23.94)$ & $3(10.34)$ & $>0.05$ \\
benign tumors & $3(4.23)$ & $10(34.48)$ & $<0.01$ \\
malignant tumors & $0(0.00)$ & $5(17.24)$ & $<0.01$ \\
hyperplastic polyp & $0(0.00)$ & $1(3.45)$ & $>0.05$ \\
\hline
\end{tabular}

\section{Discussions}

At present, colonic adenoma-colon cancer sequential theory has been got approval of the broad masses of medical workers. So the judgment of the properties of colonic polyps is of great significance for the secondary prevention of colon cancer [2]. In this study, there are 100 cases of colon polyps, 61 cases of adenomatous polyp (61.00\%), in the highest proportion of all pathological findings. Elderly group share is greater than the young and middle-aged group, and the difference is statistically significant $(\mathrm{P}<0.05)$. Colon adenomas are prone to progression to colon cancer. The elderly patients have high incidence of malignant tumor. The results of this study are consistent with this. In the young and middle-aged group, adenomatous polyp proportion is the highest, so for the young and middle-aged colonoscopy examination, revealed polypoid lesions should also be cautious; conventional endoscopic biopsy to understand the polyp property is very important. Inflammatory polyp in young and middle-aged group is greater than the elderly group, and the difference is statistically significant $(\mathrm{P}<0.05)$. This may be related to the diameter of colonic polyps in young and middle-aged group that was smaller than that of elderly group, and the larger diameter polyps are more likely to be malignant. In the elderly group, the proportion of malignant tumor was greater than that of young and middle-aged group, but there is no statistically significant difference. This may be associated with the selection of polyps in EMR and ESD operations, because endoscopic polyp morphology changes obviously and large diameter cannot be done by EMR or ESD operation patients to surgical treatment. These cases data are not included in the research. In a word, for endoscopic colonic polyps that can be treated by EMR and ESD operation, higher proportion of inflammatory polyp in young and middle-aged group and higher proportion of adenomatous polyp in elderly group, need to be mindful for cancerous possibility.

Among 100 cases of colon polyps of this study, the implementation of EMR operation is 71 cases, and there are 29 cases with ESD operation. Adenomatous polyp implemented EMR ratio is greater than the ESD operation, and the difference is statistically significant $(\mathrm{P}<0.01)$. Adenomatous polyp includes tubular adenoma, villous adenoma and tubular-villous adenoma. Among them, villous adenoma is the most likely to be malignant tumor, followed by tubular-villous adenoma. Tubular adenoma has the lowest probability of malignancy. In this 
study, tubular adenoma is the highest proportion, and the polyps' diameter which uses EMR operation was smaller than the polyps which use ESD operation, with small malignant probability. Hence, the proportion of adenomatous polyp in EMR operation is greater than the ESD operation. Similar to this principle, in the tumor diseases, whether it is benign or malignant tumor, implementation of ESD operation proportion is greater than the implementation of EMR operation, and the difference is statistically significant $(\mathrm{P}<0.01)$. At present, with the rapid development of digestive endoscopy technology, EMR and ESD operation in the treatment of digestive tract polyps play an increasingly important role. Studies from Huang Jin have shown that EMR and CIEMR (conventional endoscopic mucosal resection with circumferential incision) have a good effect in the treatment of rectal benign tumor [3]. Some scholars through retrospective study pointed out that: in the complex colon polyps, $75 \%$ of the patients have EMR operation treatment alternative to surgical treatment [4]. In the case of 21 months infants, who have huge gastric hyperplastic polyp, there is successful application of ESD removing polyps, and another study shows that ESD is used in esophageal verrucous malignant tumor resection [5] [6]. Related studies show that EMR and ESD pathological examinations after operation have differences with preoperative endoscopic biopsy results, and the difference is statistically significant. The pathological examination malignant degree after EMR and ESD operation is higher than the preoperative endoscopic biopsy [7]. This suggests that preoperative endoscopic biopsy results can only be used as a reference. Complete specimens submission to pathologic examination is the gold standard in pathological diagnosis after EMR and ESD operation.

This article has many shortcomings as a retrospective study. First of all, this study is a single center retrospective study. The number of cases collected is relatively small. The number of cases in each subgroup is less and has some impact on the statistical analysis. Secondly, because the EMR and ESD operation time in Jingzhou center hospital digestive endoscopy center is short, and the accumulation of experience is limited. For some polyps with complex structure or larger diameter, surgical treatment is often recommended. This part of the case is not covered by the study. It will also have implications for this study. At the end of this article, we hope to have more scholars on the research of EMR and ESD, so as to provide more and better clinical reference for digestive endoscopy physicians.

\section{Conflicts of Interest}

The authors declare no conflicts of interest regarding the publication of this paper.

\section{References}

[1] De, C.A., Hassan, C., Mangiavillano, B., et al. (2016) Endoscopic Mucosal Resection and Endoscopic Submucosal Dissection for Colorectal Lesions: A Systematic Re- 
view. Critical Reviews in Oncology, 104, 138-155.

https://doi.org/10.1016/j.critrevonc.2016.06.008

[2] Hu, H.Y. (2013) The Cause Analysis of 590 Cases with Gastrointestinal Bleeding. Chinese Journal of Medicinal Guide, 15, 35-36.

[3] Jin, H., Lu, Z.S., Yang, Y.S., et al. (2014) Endoscopic Mucosal Resection with Circumferential Incision for Treatment of Rectal Carcinoid Tumours. World Journal of Surgical Oncology, 12, 23. https://doi.org/10.1186/1477-7819-12-23

[4] Raju, G.S., Lum, P.J., Ross, W.A., et al. (2016) Outcome of EMR as an Alternative to Surgery in Patients with Complex Colon Polyps. Gastrointestinal Endoscopy, 84, 315-325. https://doi.org/10.1016/j.gie.2016.01.067

[5] Jung, E.Y., Choi, S.O., Cho, K.B., et al. (2014) Successful Endoscopic Submucosal Dissection of a Giant Polyp in a 21-Month-Old Female. World Journal of Gastroenterology, 20, 323-325. https://doi.org/10.3748/wjg.v20.i1.323

[6] Abe, T., Kato, M., Itagaki, M., et al. (2016) Endoscopic Submucosal Dissection for an Atypical Small Verrucous Carcinoma: A Case Report. Journal of Medical Case Reports, 10, 74. https://doi.org/10.1186/s13256-016-0866-y

[7] Elsadek, H.M. and Radwan, M.M. (2015) Diagnostic Accuracy of Mucosal Biopsy versus Endoscopic Mucosal Resection in Barrett's Esophagus and Related Superficial Lesions. International Scholarly Research Notices, 2015, Article ID: 735807. 